\title{
Contribution à la méthode convergence-confinement par le principe de la similitude
}

\author{
Contribution to the characteristic lines method \\ by the principle of the similitude
}

\author{
F. CORBETTA, D. BERNAUD, D. NGUYEN MINH \\ Laboratoire de Mécanique des Solides \\ Centre commun X-ENSMP-ENPC-CNRS U.A. D0317*
}

Rev. Franç. Géotech. n 54 , pp. 5-11 (janvier 1991)

\section{Résumé}

La méthode convergence-confinement, très pratique pour le dimensionnement des tunnels soutenus dans les terrains sans effets différés, souffre cependant d'un inconvénient. La convergence acquise par la galerie au moment de la pose du soutènement, en relation avec la distance au front, reste indéterminée.

On propose une méthode simple pour obtenir de façon approchée la déformée de la paroi en fonction de la distance au front et ainsi lever une telle indétermination dans le cas d'un comportement élastoplastique parfait. Cette approximation est validée pour les facteurs de chargement courants.

A l'aide de cette méthode on examinera ensuite les pressions de soutènement et les convergences finales en fonction de la plastification du massif et de la distance au front à laquelle est posé le soutènement.

\footnotetext{
Abstract

The characteristic lines method is very useful for the design of supported tunnels in grounds with no time-dependent effects, but has however a drawback. The convergence of the tunnel when the support is installed, in relation to the distance from the front face, remains undetermined.

A simple method is proposed in order to get a precise assessment of the convergence at the wall related to the distance from the front in the case of an elastic perfectly plastic ground behaviour. This approximation is validated for the usual loading factors.

Using this method, final support pressures and final wall convergences are also investigated according to the ground plasticity and the distance from the front where support is installed.
} 


\section{INTRODUCTION}

La méthode convergence-confinement, aussi connue sous le nom de méthode des lignes caractéristiques, est une méthode de prédimensionnement des tunnels profonds soutenus représentant la nouvelle approche qui prend en compte l'interaction entre le massif et la structure, par opposition aux anciennes méthodes qui remplaçaient le massif par un système de charges agissant a priori sur le soutènement (méthodes des réactions hyperstatiques), ou qui ne considéraient pas les phases d'excavations (méthodes du solide composite).

La méthode convergence-confinement se place habituellement en symétrie axiale, en considérant une galerie de section circulaire, de rayon $\mathrm{R}$, creusée dans un massif infini, homogène, isotrope. Cette galerie est à une profondeur suffisante pour qu'on puisse négliger la variation de contrainte géostatique entre la voute et le radier. Avant l'excavation, le massif est soumis à un état initial de contraintes isotrope, caractérisé par la pression $\mathrm{P}$ qui résulte du poids des terrains sus-jacents.

L'excavation d'une telle galerie avec la prise en compte de l'effet du front de taille est un problème tridimensionnel, qui peut se ramener à l'étude d'un problème équivalent bidimensionnel en déformations planes (PANET, GUELLEC, 1974). L'effet du passage du front est alors équivalent à la décroissance d'une pression intérieure fictive $\mathrm{Pi}^{f}$ en paroi, depuis la pression initiale $\mathrm{P}$ régnant avec l'excavation, jusqu'à une pression nulle bien après le passage du front (fig. 1).

L'étude d'une section circulaire, dans un massif initialement précontraint, soumise à une pression intérieure $\mathrm{Pi}$ en paroi, permet alors de simuler l'effet du passage du front, par la pression intérieure fictive $\mathrm{Pi}^{\mathrm{t}}$, et l'effet d'un soutènement posé à une distance d du front, par la pression de soutènement $\mathrm{Pi}^{\mathrm{s}}$.

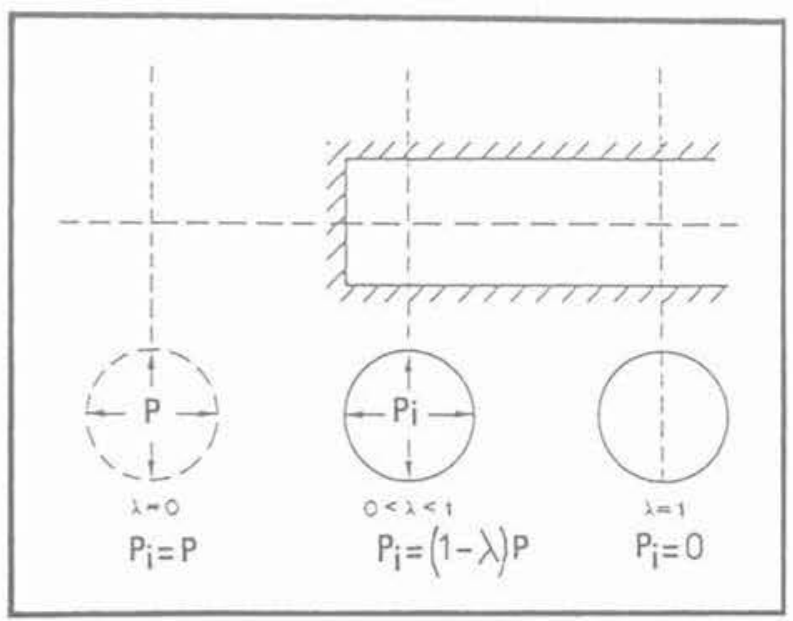

Fig. 1. - Principe de la méthode Convergence-Confinement (d'après PANET, GUENOT, 1982).

Fig. 1. - Characteristics Lines method principle (from PANET, GUENOT, 1982).
Ceci permet de définir les deux courbes caractéristiques, du massif et du soutènement, qui relient les pressions intérieures respectives $\mathrm{Pi}^{f}$ et $\mathrm{Pi}^{5}$ à la convergence radiale relative en paroi $u / R$. Le point d'équilibre final, une fois que le front de taille s'est suffisamment éloigné, est donné par l'intersection de ces deux courbes caractéristiques respectives (fig. 2).

En élasticité les courbes caractéristiques du massif et du soutènement sont des droites puisque pour le massif : $\mathrm{u} / \mathrm{R}=\left(\mathrm{P}-\mathrm{Pi}^{i}\right)(1+\nu) / \mathrm{E}$ et pour le soutènement : $\mathrm{Pi}^{\mathrm{s}}=\mathrm{K}\left(\mathrm{u} / \mathrm{R}-\mathrm{u}_{0} / \mathrm{R}\right)$, E et $\nu$ étant le module d'Young et le coefficient de Poisson du massif, $\mathrm{K}$ étant la rigidité du soutènement. Par contre, la courbe caractéristique d'un massif élastoplastique n'est plus une droite et est obtenue à l'aide de solutions explicites (BROWN et al., 1983 ; NGUYEN MINH et al., $1979, \ldots)$ ou de modèles numériques unidimensionnels (PANET et al., 1974,...).

Tout le problème consiste à déterminer la convergence $\mathrm{u}_{0} / \mathrm{R}$ acquise en paroi de la galerie au moment de la pose du soutènement à une distance $\mathrm{D}$ du front ; D étant la distance au front exprimée en rayons $\mathrm{D}=\mathrm{d} / \mathrm{R}$.

Pour déterminer $u_{0} / R$, la méthode convergenceconfinement utilise le taux de déconfinement $\lambda$ défint par $\mathrm{Pi}^{f}=(1-\lambda) \mathrm{P}$ avec $\lambda$ croissant de 0 à 1 pour simuler l'avance de l'excavation. Ce taux $\lambda$ dépend de la distance au front et une fois $\lambda$ déterminé, on remonte à $\mathrm{u}_{0} / \mathrm{R}$ par l'intermédiaire de la courbe caractéristique du massif. Dans le cas élastique, $\lambda$ est égal au rapport des convergences respectivement, intermédiaire à une distance $\mathrm{d}$ du front, et finale très loin du front. Par contre dans le cas élastoplastique ceci cesse d'être vérifié.

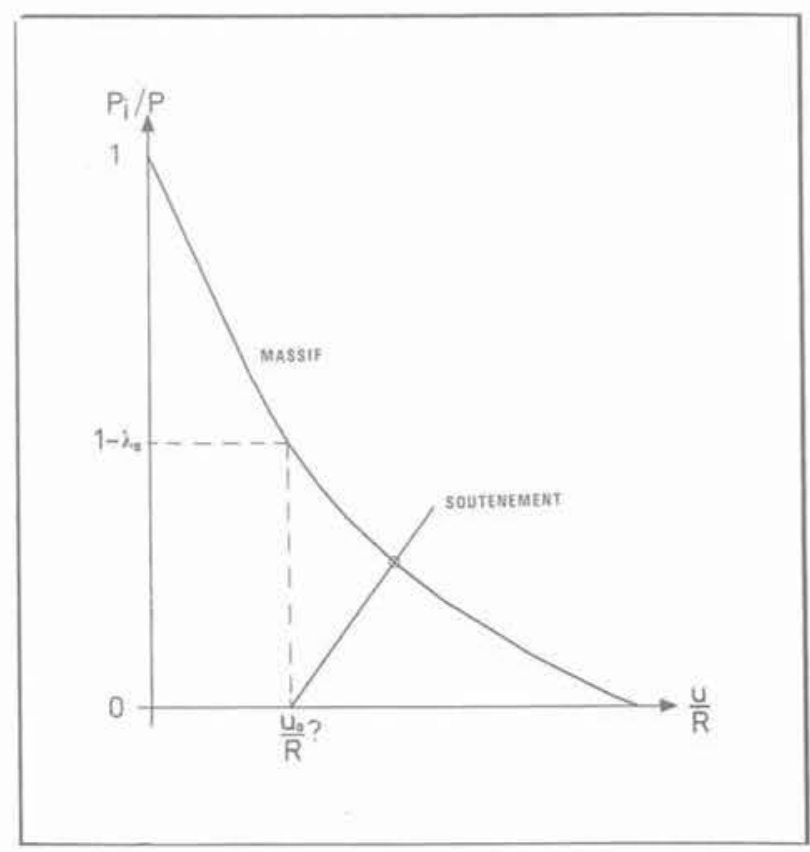

Fig. 2. - Courbes caractéristiques du massif et du soutènement.

Fig. 2. - Ground and support characteristic lines. 
L'AFTES (Tunnels et Ouvrages Souterrains, 1983, $\mathrm{n}^{\circ} 59$, p. 230) propose une table de décision pour la relation entre ce taux de déconfinement $\lambda$ et la distance au front à laquelle est installé le soutènement. Mais cette table ne concerne qu'un comportement élastoplastique purement cohérent du massif et de plus, une certaine imprécision est à noter aux alentours du front : à chaque distance de pose correspond une plage de valeurs. Il est même dit que "si le niveau de précision requis ou si la plastification sont trop importants on deura recourir à l'utilisation d'un modèle numérique.

Nous avons donc tenté de nous affranchir de l'étape précédente, en cherchant une relation plus directe entre la distance au front et la convergence en paroi correspondante par une étude détaillée des profils de convergence.

\section{MODÉLISATION ET CALCULS}

Compte tenu des hypothèses faites précédemment, un modèle numérique aux éléments finis a été utilisé en axisymétrie pour l'étude d'une galerie de section circulaire avec front de taille, située dans un massif initialement contraint.

Le comportement du massif est supposé élastoplastique parfait avec des critères de plasticité de type Von Mises et Drucker-Prager. De plus l'incompressibilité élastique du massif est supposée $(\nu=0,5)$, ce qui ne nuit pas à la généralité des calculs, puisqu'on sait que le coefficient de Poisson influe assez peu sur la convergence en paroi d'un massif élastoplastique, mais simplifie les calculs analytiques comparatifs et permet une correspondance simple entre les critères de Von Mises et Tresca d'une part, et de Drucker-Prager et Coulomb dans le cas de l'incompressibilité plastique.

Le modèle numérique utilisé était constitué de 2400 nœeuds correspondant à 600 quadrilatères à 9 nœuds d'interpolation quadratique (élément Q9). Le rapport du rayon extérieur du modèle au rayon intérieur $\mathrm{R}$ de la galerie était de 30 . Le modèle s'étendait, dans laxe longitudinal, entre - 20R en amont du front et $40 \mathrm{R}$ en aval du front. Une discrétisation fine autour du front de taille a été faite: la totalité du modèle se composait de 80 tranches radiales dans l'axe longitudinal dont plus de la moitié entre $-2 \mathrm{R}$ et $2 \mathrm{R}$ (fig. 3)

Les données étaient les suivantes $\mathrm{P}=4,4 \mathrm{MPa}$, $E=1430 \mathrm{MPa}, \nu=0,498$. On a envisagé un comportement de type élastoplastique parfait défini par une cohésion $C$, un angle de frottement $\phi$ et un coefficient de dilatance $\beta$. Il a aussi été utilisé le facteur de stabilité Ns égal au rapport de la pression initiale $\mathrm{P}$ au "seuil de plasticité » $\mathrm{R}_{\mathrm{c}} / 2, \mathrm{R}_{\mathrm{c}}$ étant la résistance du massif à la compression simple:

$$
\begin{gathered}
N_{s}=\frac{2 P}{R_{c}} \text { avec } R_{c}=\frac{2 C \cos \phi}{1-\sin \phi} \\
\text { (il } y \text { a donc plasticité si } N_{s}>1 \text { ) }
\end{gathered}
$$

On a utilisé la méthode dite des a contraintes initiales \# avec un algorithme implicite pour résoudre le problème élastoplastique non linéaire et la tolérance de la solution sur le critère de plasticité était de $0,1 \%$.

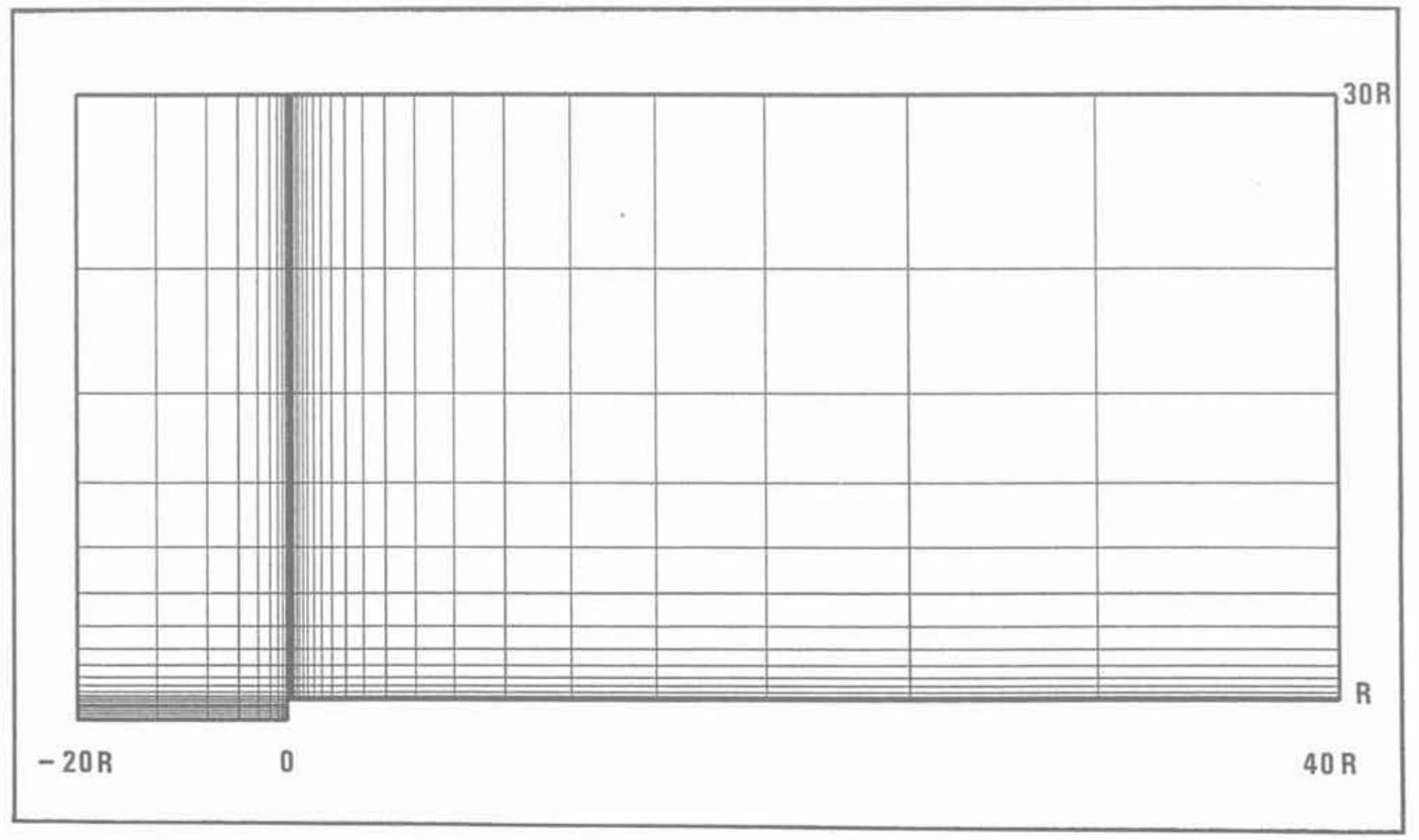

Fig. 3. - Maillage du modèle.

Fig. 3. - Madel mesh. 
Les calculs ont permis d'aboutir aux profils de convergence radiale relative $u / R$ en paroi en fonction de la distance $D$ au front de taille (fig. 4), ceci pour différents types de comportement élastoplastique parfait ainsi que pour le cas élastique (fig. 4) $(\mathrm{Ns}=1)$.

La vue de ces profils suggère une transformation géométrique qui traduirait la similitude qu'on peut observer sur ces figures, et qui permettrait de déduire les profils élastoplastiques à partir du seul profil élastique. Nous avons donc testé lhomothétie, qui est la transformation la plus simple qui vienne ici à l'esprit.
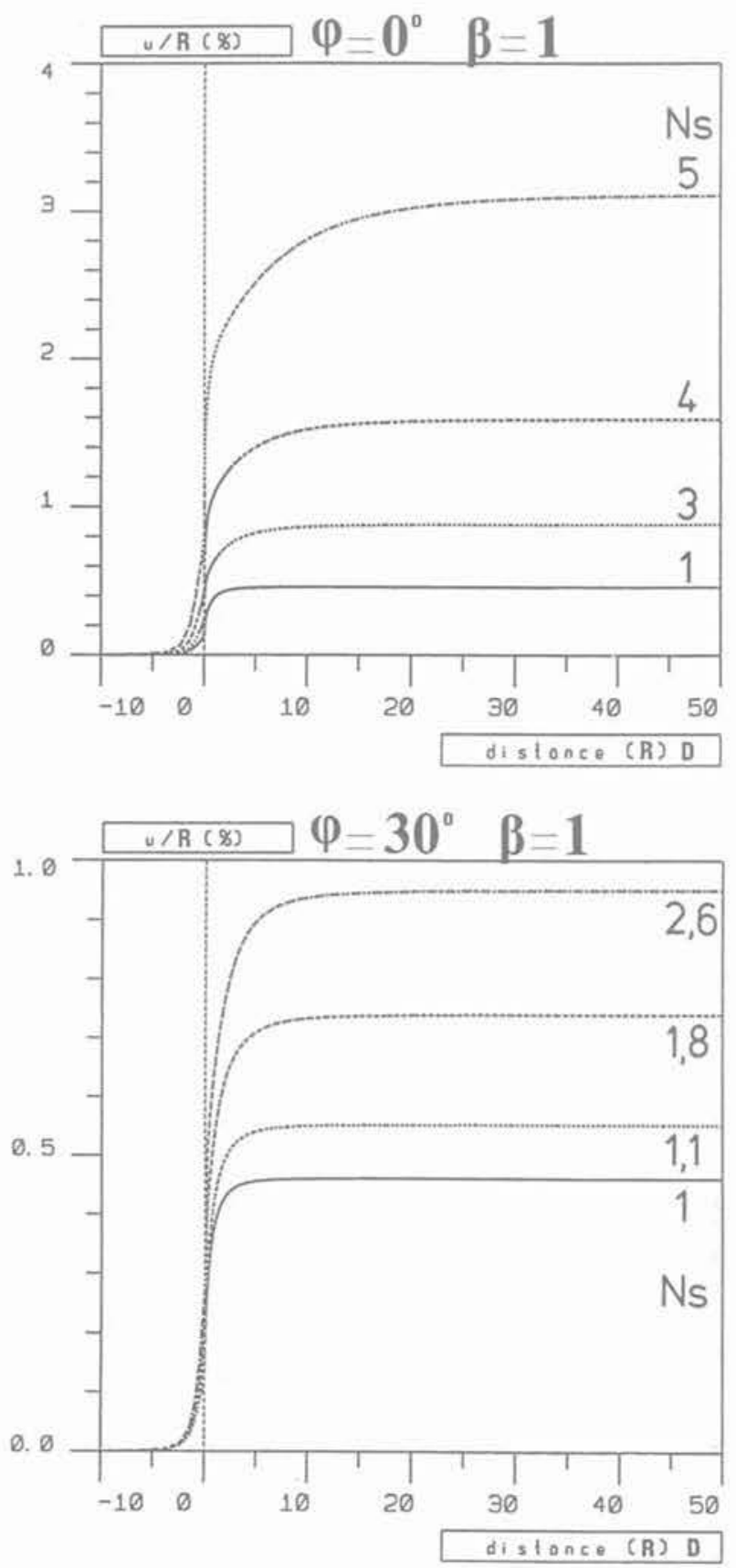

Fig. 4. - Profils de convergence en paroi $u / R$ en fonction de la distance au front $D$ selon le taux de chargement Ns. Fig. 4. - Profiles of wall convergence $U / R$ versus the distance from the front face $D$ according to the loading factor Ns.
Cette homothétie est définie par son centre qu'on prendra à l'origine, au front de taille actuel, et par son rapport $\chi$ qui sera défini comme le rapport des $\mathrm{u} / \mathrm{R}$ en paroi, respectivement plastique et élastique, pour une section très éloignée du front. Il s'agit alors de vérifier que les profils de convergence obtenus par les calculs E.F. satisfont bien à cette homothétie.

Les profils élastoplastiques obtenus par les calculs ont été ainsi comparés avec ceux obtenus par la transformation du profil élastique (fig. 5).

Il est à noter qu'un bon calage est obtenu même pour le cas le plus défavorable qui concerne le milieu purement cohérent avec $\mathrm{Ns}=5$, qui est considéré comme un cas limite dans la recommandation de l'AFTES citée: dans ce cas, la précision devient moins bonne près du front avec une sous-estimation maximale sur la convergence qui reste tout de même inférieur à $10 \%$. Pour les cas courants (Ns < 5 ) l'approximation est très satisfaisante.

\section{DÉTERMINATION PRATIQUE DE LA CONVERGENCE A LA POSE}

\section{Méthode graphique}

La méthode graphique est représentée sur la figure 6 .

La droite caractéristique du massif élastique O'A est connue. La courbe caractéristique du massif élastoplastique O'C est obtenue par une solution analytique ou par un modèle numérique unidimensionnel. Le rapport des convergences finales, plastique et élastique, du massif détermine immédiatement le rapport $\chi=O C / O A$ de l'homothétie. Maintenant le profil longitudinal de convergence élastique va permettre de déterminer $\mathrm{u}_{0} / \mathrm{R}$ grâce à l'homothétie.

Le soutènement est installé à une distance $\mathrm{D}$ du front. La première étape consiste à se ramener à une distance $\mathrm{D} / \chi$ et de déterminer la convergence radiale élastique correspondante (point $\alpha$ ). Le point $\alpha$ et le centre de lhomothétie $\mathrm{O}$ définissent une droite sur laquelle se trouve le point homothétique $\gamma$ correspondant à une distance de pose D ; le rapport des segments $\mathrm{O} \gamma$ et $\mathrm{O} \alpha$ étant égal à $\chi$. L'ordonnée du point $\gamma$ correspond à la convergence cherchée et il est alors possible de tracer la droite caractéristique du soutènement.

\section{Méthode analytique}

Le profil élastoplastique a été tracé en pointillés pour bien montrer que l'homothétie permet de l'approximer, mais son tracé n'est plus nécessaire. De même. si on possède une formule explicite pour le profil élastique $u / R=f(D)$ alors on peut aussi se dispenser de tracer le profil élastique puisque l'homothétie donne directement pour le cas élastoplastique $\mathrm{u}_{0} / \mathrm{R}=$ $\chi \mathrm{f}(\mathrm{D} / \chi)$.

Un très bon calage a été obtenu avec l'expression suivante dans le cas élastique:

$$
\frac{\mathrm{u}}{\mathrm{R}}=\frac{\mathrm{u}_{\mathrm{e}}}{\mathrm{R}}\left[0,29+0,71\left[1-\exp \left(-1,5 \cdot \mathrm{D}^{0,7}\right)\right]\right\}
$$



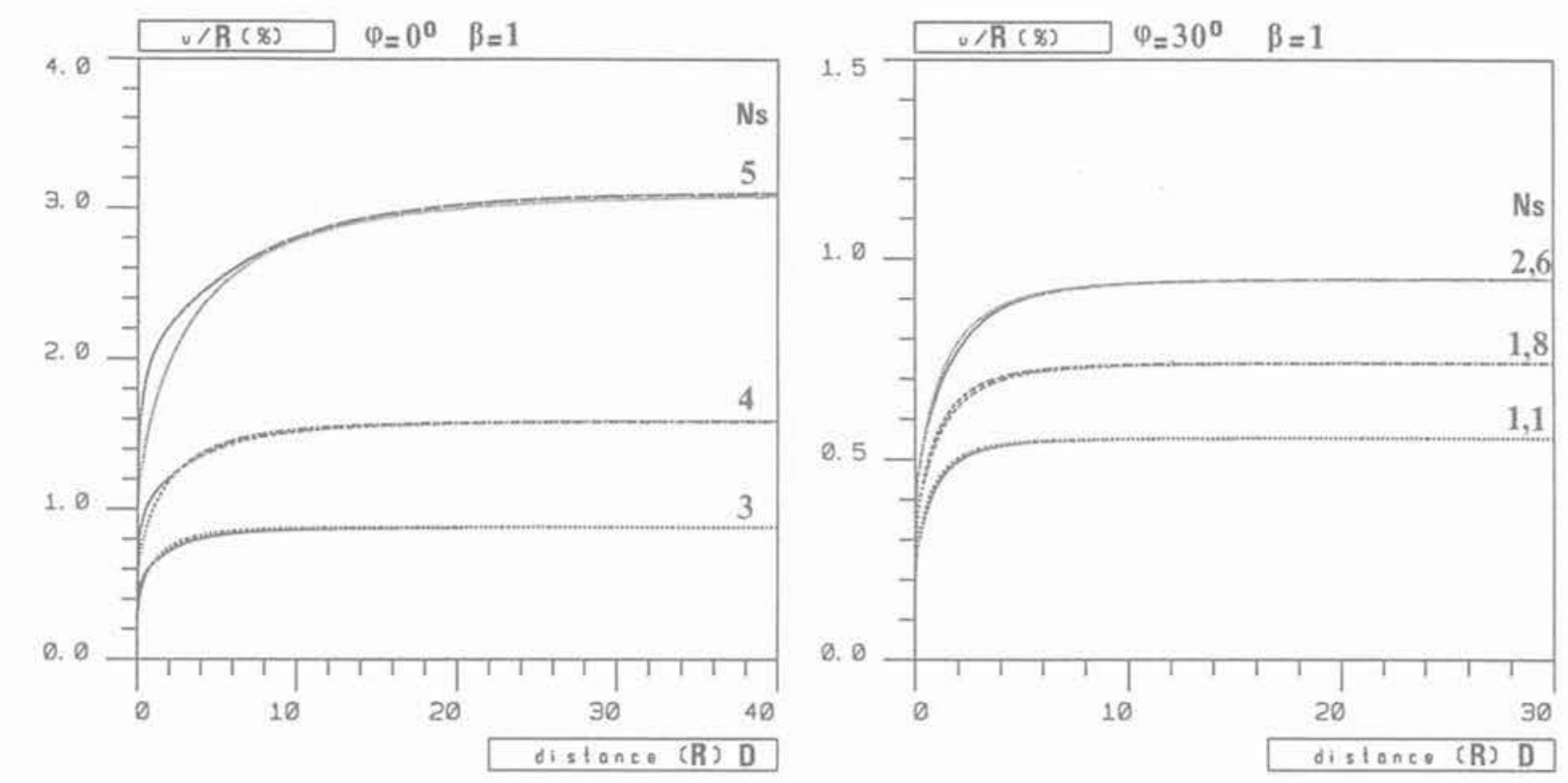

Fig. 5. - Comparaison des profils de convergence - Eléments Finis/Homothétie.

Fig. 5. - Comparison of the convergence profiles - Finite Elements/Similarity.

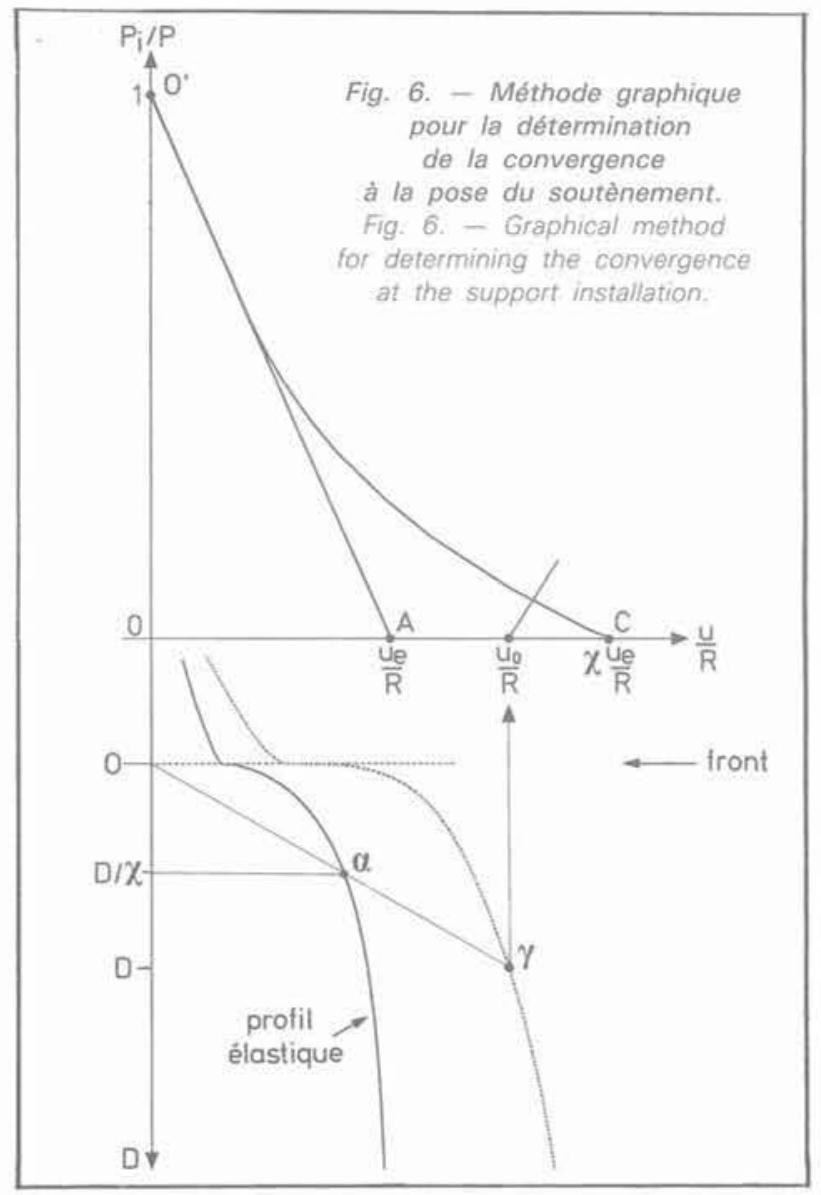

avec :

$\frac{\mathrm{u}_{\mathrm{e}}}{\mathrm{R}}=\frac{1+\nu}{\mathrm{E}} \mathrm{P}$ (convergence finale élastique)

et $\mathrm{D}=\frac{\mathrm{d}}{\mathrm{R}}$ (distance au front).

Cette expression détermine la convergence radiale élastique en paroi de la galerie, à une distance D du front. L'homothétie détermine ensuite la convergence élastique correspondant à cette même distance D du front où est installé le soutènement:

\begin{tabular}{|ll|}
\hline élastique & élastoplastique $\chi$ \\
$\frac{\mathrm{u}}{\mathrm{R}}=\mathrm{f}(\mathrm{D})$ & $\frac{\mathrm{u}}{\mathrm{R}}=\chi \mathrm{f}\left(\frac{\mathrm{D}}{\chi}\right)$ \\
\hline
\end{tabular}

\section{Remarque}

Le profil de convergence élastoplastique n'est autre que le profil élastique pour une galerie de rayon $\chi \mathrm{R}$; la plasticité se traduit donc pour le profil des convergences par une augmentation fictive du rayon de la galerie, dans le même milieu élastique. On retrouve l'idée de rayon équivalent évoquée par KAISER (1981). 


\section{Conclusions}

La convergence $u_{0} / \mathrm{R}$, point de départ du tracé de la droite caractéristique du soutènement élastique, dépend donc de la plastification du massif et est donc différente selon chaque facteur de stabilité Ns considéré ; en particulier s'il y a déjà plastification à la pose du soutènement on ne peut pas prendre le même point de départ pour le cas élastique et le cas élastoplastique. Cette distinction selon la plastification tend à réduire les écarts sur les pressions de soutènement finales entre l'élasticité et l'élastoplasticité, voire quelquefois à les inverser.

\section{EXEMPLES D'APPLICATION}

A titre d'exemple, nous appliquons la méthode au calcul de la pression de soutènement pour un tunnel dans un massif incompressible soumis à une pression géostatique $\mathrm{P}$, et dont le comportement est élastoplastique parfait, défini par un module d'Young $\mathrm{E}$ et un critère de Tresca ou de Coulomb, de frottement interne $\phi$. Le soutènement est supposé élastique linéaire, de rigidité $\mathrm{K}$.

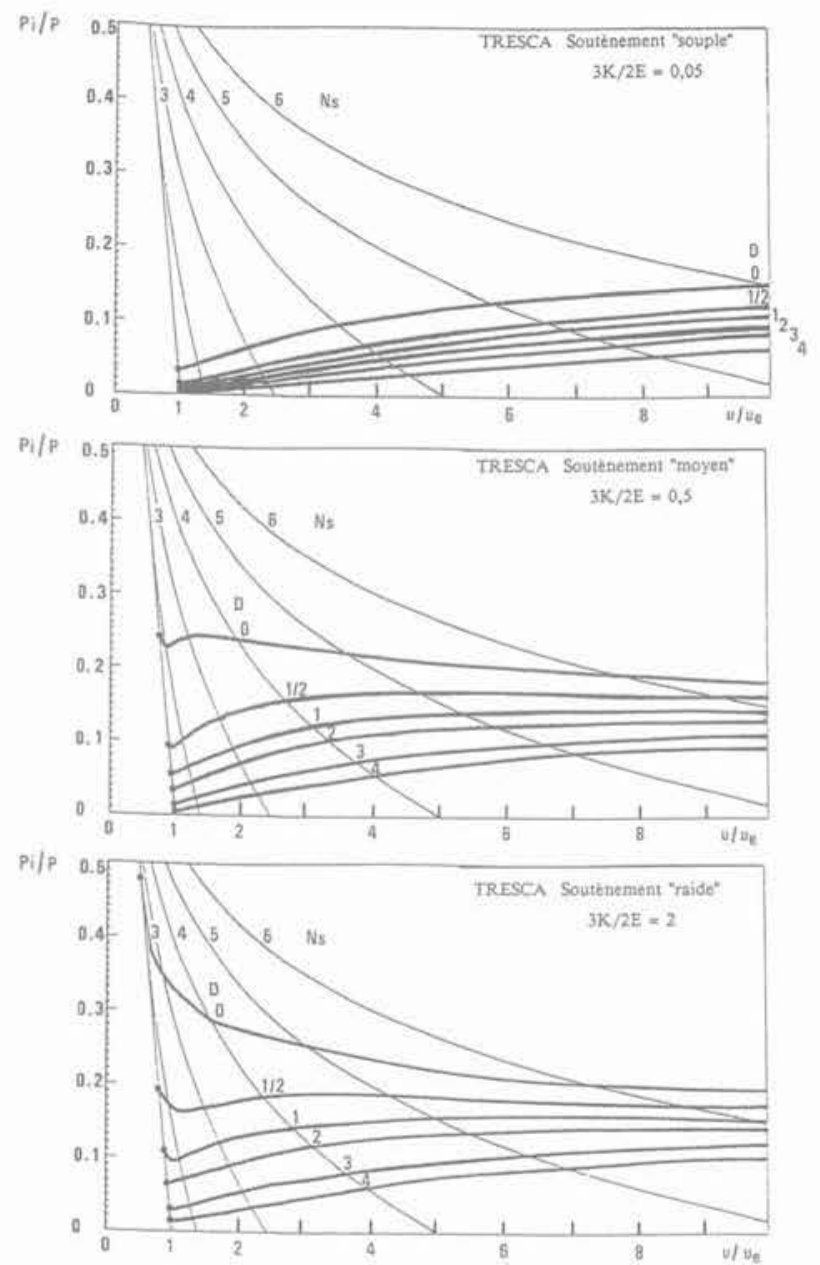

Les résultats sont donnés sous forme de lieu des points d'équilibre final sur les courbes caractéristiques pour diverses rigidités $\mathrm{K}$ du soutènement. Les convergences ont été normées par rapport à la convergence finale $u_{e} / R=3 P / 2 E$ du milieu élastique de même module $\mathrm{E}$, et les pressions intérieures par rapport à la pression initiale $\mathrm{P}$. Ceci fait que la pente de la droite caractéristique du soutènement passe de $\mathrm{K}$ à $k_{\mathrm{s}}=3 \mathrm{~K} / 2 \mathrm{E}$ qui varie pour les soutènements courants de 0.05 à 2 ; du boulonnage à l'anneau en béton dans un massif de module d'Young $1500 \mathrm{MPa}$ (HOEK, BROWN, 1980).

L'étude porte sur différentes valeurs de la distance de pose $D$ et du facteur de stabilité Ns (fig. 7).

On constate que pour un soutènement suffisamment raide $\left(k_{s}=2\right)$, le taux de pression de soutènement $\mathrm{Ps} / \mathrm{P}$ peut diminuer lorsque le taux de charge Ns augmente; ce phénomène a lieu pour les faibles valeurs de Ns ou en deçà d'une certaine distance relative $D$ du front, et son ampleur est accentuée pour le milieu de Coulomb.

Par contre, pour le revêtement souple $\left(k_{\mathrm{s}}=0,05\right)$, le taux de pression de soutènement a une évolution
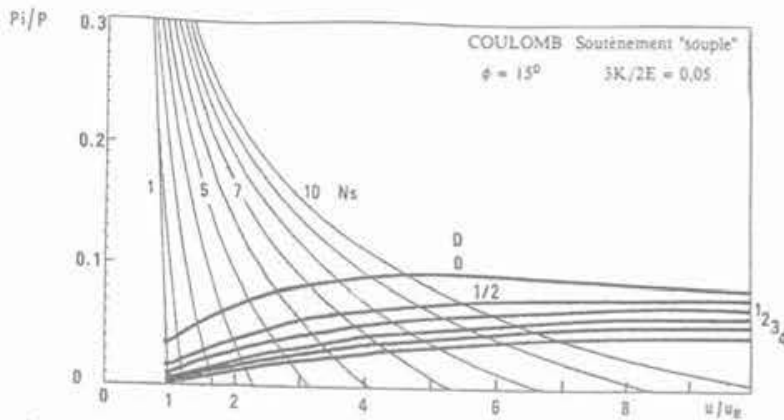

Pitp

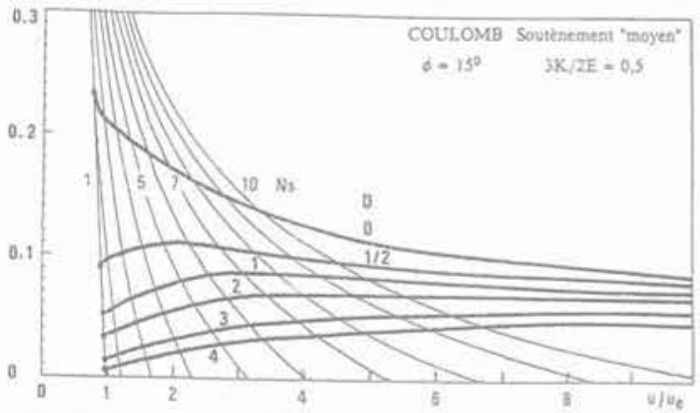

$\mathrm{Pi} / \mathrm{p}$

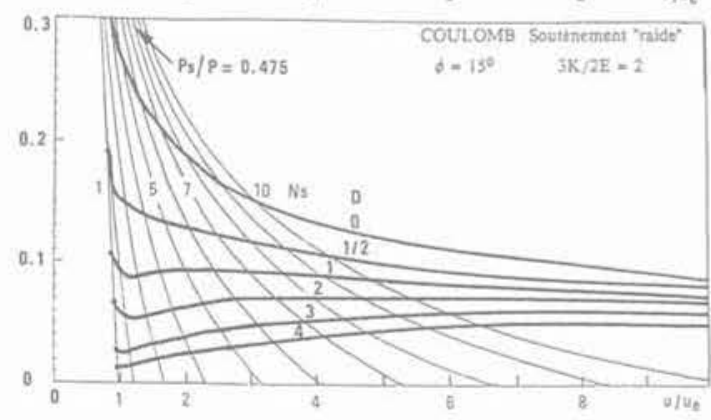

Fig. 7. - Lieu des pressions finales de soutènement Ps et convergences finales u/R selon la distance au front $D$ et le taux de chargement Ns.

Fig. 7. - Curves of the final support pressures PS and final convergences u/P according to the distance from the front D and the loading factor Ns, 
classique, il augmente avec Ns. Entre ces deux cas extrême, on a des comportements intermédiaires selon les valeurs de $\mathrm{D}$ et de l'angle de frottement interne $\phi$ du matériau de Coulomb, par exemple le passage de $\mathrm{Ps} / \mathrm{P}$ par un minimum, ou inversement par un maximum, ou parfois les deux.

Ces résultats mettent ainsi en défaut l'idée classique selon laquelle l'augmentation du taux de plasticité Ns se traduit toujours par une augmentation de la charge sur le revêtement.

\section{CONCLUSION}

Nous avons présenté une procédure simple pour déterminer, dans le cas d'un comportement élastoplastique parfait, la convergence acquise par une galerie au moment de la pose du soutènement, en relation avec la distance au front de taille. Cette procédure est fondée sur la propriété de similitude de la déformée de la galerie par rapport à sa déformée " fictive " élastique; elle apporte un complément précieux à la méthode convergence-confinement, et permet de s'affranchir de la détermination du taux de déconfinement $\lambda$.

Comme exemple d'application, nous avons étudié de façon systématique l'influence de la distance de pose du revêtement dans le cas du matériau élastoplastique parfait de Tresca et de Coulomb. Cette étude a permis notamment de mettre en évidence un phénomène original, a priori surprenant, à savoir, pour une profondeur donnée, la diminution dans certains cas, surtout près du front, de la pression sur le revêtement lorsque la plastification du terrain augmente; ce résultat suggère que la pression sur le revêtement peut être interprétée comme une pression mobilisable du terrain, derrière le front de taille, et que sa valeur reste limitée : c'est le terrain élastique qui offre le maximum de pression mobilisable, près de $70 \%$ de la pression géostatique, pour un revêtement infiniment rigide posé immédiatement derrière le front.

Bien entendu, ces résultats ne sont valables que dans le cadre du type de comportement retenu, essentiellement non visqueux ; on ne peut pas par exemple, déduire sans précautions les pressions finales sur le revêtement en se basant seulement sur les caractéristiques à long terme d'un matériau doté d'effets différés.

La méthode s'applique au calcul de prédimensionnement des revêtements de tunnels avec la méthode convergence-confinement, mais aussi à l'interprétation des convergences mesurées in situ où elle constitue une alternative avantageuse à d'autres méthodes em. piriques déjà proposées.

Il reste à savoir si la propriété de similitude des déformées du tunnel en arrière du front reste vraie dans le cas d'un comportement élastoplastique plus complexe, incluant notamment de l'écrouissage. Nous sommes persuadés qu'il en est bien ainsi dans le cadre de l'approximation consentie, sous réserve de vérification; dans ce cas, la propriété aurait un caractère " universel », autrement, elle serait réservée à une certaine classe de matériaux et servirait de référence pratique pour distinguer d'autres types de comportement.

\section{BIBLIOGRAPHIE}

AFTES (1983), Recommandations sur l'emploi de la méthode convergence-confinement. Tunnels \& Ouvrages Souterrains, n०59, sept.-oct. 1983.

BROWN E.T., BRAY J.W., LADANYI E., HOEK E. (1983), Ground Response Curves for Rock Tunnels. Journal of the Geotechnical Engineering Division, ASCE, Vol. 109, pages 15-39.

CORBETTA F. (1990),, Calculs analytiques et numériques de tunnels profonds. Thèse, EMSMP, octobre 1990.

HOEK E., BROWN E.T. (1980), Underground Excavations in Rock. The Institution of Mining and Metallurgy, London, 1980.

KAISER P.K. (1981), A New Concept to Evaluate Tunnel Performance-Influence of Excavation Procedure. Proceedings of the $22^{\text {nd }}$ US Symposium on Rock Mechanics, 1981, MIT.

NGUYEN MINH D., BEREST P. (1979), Etude de la stabilité des cavités souterraines avec un modèle de comportement élastoplastique radoucissant. Proceedings of the $4^{\text {th }}$ International Congress of Rock Mechanics, 1979, Montreux.

PANET M., GUELLEC P. (1974), Contribution au problème de l'étude du soutènement d'un tunnel derrière le front de taille. Proceedings of the $3^{\text {rd }}$ International Congress of Rock Mechanics, 1974, Denver.

PANET M., GUENOT A. (1982), Analysis of Convergence behind the Face of a Tunnel. Proceedings of the ISRM Symposium : Tunnelling 82', 1982, Brighton. 